\title{
Orthonormalization of Radiation Modes in Effective Resonator Model of Dielectric Multilayer Structure
}

\author{
A. RUDZIŃSKI* \\ Institute of Microelectronics and Optoelectronics \\ Warsaw University of Technology \\ Koszykowa 75, 00-665 Warszawa, Poland
}

(Received May 9, 2007)

In this paper, the orthonormal mode set is derived from electromagnetic field distributions of a dielectric multilayer structure, which have been obtained using the effective resonator model, presented in Acta Phys. Pol. A 111, 323 (2007)

PACS numbers: 42.70.Qs

\section{Introduction}

The problem of electromagnetic field in a dielectric multilayer structure, in particular radiation modes, has been already studied in other papers and books, e.g. [1-4]. In these works, there has been adopted an approach, in which the electromagnetic field of a radiation mode is constructed as a superposition of two plane waves incident on the structure from opposite directions. The relation between the amplitudes of these waves is determined by the demand for the field to form standing waves in the regions outside the structure in the direction perpendicular to the layers, so that no power flow occurs in this direction. The requirement of two sources for only one mode can be a bit confusing, and there is a problem with this approach, when all the refractive indices become equal to 1: in this case the solution should be just a plane wave, but with two sources radiating in opposite directions it is impossible.

This inconvenience is not present in the effective resonator model. This model is a tool, which has been proposed for modeling photonic crystals [5]. It is based on a simple concept and has a clear physical interpretation. In the model, the source of the radiation is assumed to be inside the structure, and the field distributions are found by solving the boundary problem for only one source plane wave. In [6] it has been shown how this model can be used to obtain electromag-

*e-mail: a.rudzinski@elka.pw.edu.pl 
netic field distributions of a one-dimensional photonic crystal. The model is not bound by periodicity of the structure, and the results presented therein remain valid in a general case of a dielectric multilayer structure. However, the obtained field distributions are not orthogonal, and their scalar products are unknown. To render the model fully complete, it is necessary to transform these distributions into a set of orthonormal modes. In this paper, orthonormalization of radiation modes is performed. This paper is organized as follows. The considered structure is introduced in Sect. 2 and Sect. 3 contains a brief description of notation. The orthonormalization of electric field distributions is presented in Sect. 4, while Sect. 5 is devoted to magnetic field in the structure. A few helpful formulae can be found in the Appendix.

\section{Definition of the structure}

The considered dielectric multilayer structure (Fig. 1) is built of a finite number of dielectric layers, with the $j$-th layer having width $L_{z}^{(j)}$ and refractive index $n_{(j)}$. The distribution of refractive index in the whole structure is therefore

$$
n(\boldsymbol{r})=n_{(j)} \chi_{j}(z)
$$

where $\chi_{j}(z)$ is the characteristic function of the $j$-th layer, defined as:

$$
\chi_{j}(z)= \begin{cases}1, & z \in j \text {-th layer } \\ 0, & \text { otherwise }\end{cases}
$$

Magnetic permeability of all materials is assumed to be constant and equal to magnetic permeability of vacuum $\mu_{0}$.

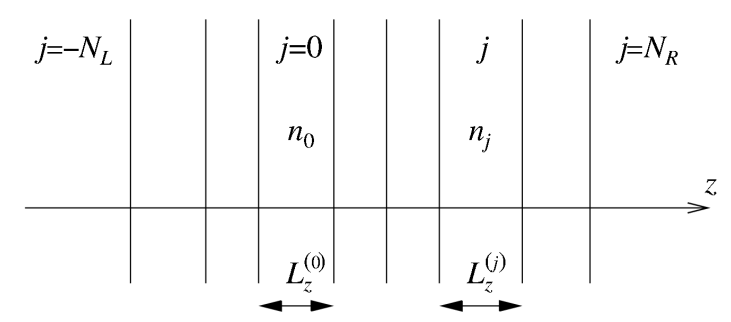

Fig. 1. A dielectric multilayer structure.

The layer, in which the source of radiation is located, has the index $j=0$. The semi-infinite regions on the "right" and "left" side of the structure have $j=N_{\mathrm{R}}$ and $j=-N_{\mathrm{L}}$, respectively, and they are considered to be layers of the structure as well.

\section{Adopted notation}

In the paper, notation $\boldsymbol{k}_{*}$ denotes the wave vector of "reflected" plane wave, in the adopted coordinate system

$$
\boldsymbol{k}_{*}=k_{x} \boldsymbol{e}_{x}+k_{y} \boldsymbol{e}_{y}-k_{z} \boldsymbol{e}_{z}
$$


$\boldsymbol{k}^{(j)}$ is the wave vector of plane wave in the $j$-th layer $\left(\boldsymbol{k}^{(0)} \equiv \boldsymbol{k}\right)$ :

$$
\boldsymbol{k}^{(j)}=k_{x} \boldsymbol{e}_{x}+k_{y} \boldsymbol{e}_{y}+k_{z}^{(j)} \boldsymbol{e}_{z} .
$$

The component of wave vector $\boldsymbol{k}$, which is parallel to the interfaces between the layers, is conserved, its length

$$
k_{\|}=\sqrt{k_{x}^{2}+k_{y}^{2}}
$$

is the same in every layer. The perpendicular component $k_{z}^{(j)}$ depends on the layer

$$
k_{z}^{(j)}=k_{z} \sqrt{1+\frac{n_{(j)}^{2}-n_{(0)}^{2}}{c^{2} k_{z}^{2}} \omega_{k}^{2}} .
$$

In case of negative expression under the square root, the sign of the root has to be chosen so that the amplitude of the wave diminishes with the distance to the source of radiation, i.e. the same as the sign of $j$. The angular frequency $\omega_{k}$ is constant for each $\boldsymbol{k}$ and given by

$$
\omega_{k}^{2}=\frac{c^{2}\left(\boldsymbol{k}^{(j)}\right)^{2}}{n_{(j)}^{2}}=\frac{c^{2} \boldsymbol{k}^{2}}{n_{(0)}^{2}} .
$$

It follows from $(6)$ that $k_{z}^{(j)}$ is either real or purely imaginary. The well-known classification of modes, based on $k_{z}^{\left(-N_{\mathrm{L}}\right)}$ and $k_{z}^{\left(N_{\mathrm{R}}\right)}$, is therefore:

1. Radiation modes - being of interest in this paper - for which both $k_{z}^{\left(-N_{\mathrm{L}}\right)}$ and $k_{z}^{\left(N_{\mathrm{R}}\right)}$ are real.

2. Substrate modes - for which exactly one of $k_{z}^{\left(-N_{\mathrm{L}}\right)}$ and $k_{z}^{\left(N_{\mathrm{R}}\right)}$ is real.

3. Guided modes - for which both $k_{z}^{\left(-N_{\mathrm{L}}\right)}$ and $k_{z}^{\left(N_{\mathrm{R}}\right)}$ are purely imaginary.

Vector $\boldsymbol{r}^{(j)}$ is used to denote position translated to the local coordinate system of the $j$-th layer. In this system, coordinates $x$ and $y$ are the same as in the global coordinate system, and the coordinate $z^{(j)}$ runs from 0 to $L_{z}^{(j)}$ in the same direction as the $z$ axis of the global coordinate system. The exception is the region $j=-N_{\mathrm{L}}$, in which $z^{\left(-N_{\mathrm{L}}\right)}$ runs from $-\infty$ to 0 .

$\rho_{\epsilon}(\boldsymbol{k})$ is the so-called mode spectrum (see [5]), a function of wave vector $\boldsymbol{k}$ and polarization $\epsilon$, calculated for the layer containing the source of radiation. For the presented calculations, the following properties of mode spectrum of the considered structure are important: it does not depend on the sign of any component of $\boldsymbol{k}$, in particular

$$
\rho_{\epsilon}(\boldsymbol{k})=\rho_{\epsilon}\left(\boldsymbol{k}_{*}\right)
$$

and

$\rho_{\epsilon}(\boldsymbol{k})>0$ for radiation modes.

\section{Electric field}

Electric field in the structure is given by [6]:

$$
\boldsymbol{E}(\boldsymbol{r}, t)=\sum_{\epsilon} \int \mathrm{d}^{3} k A_{k \epsilon}(t) \boldsymbol{\psi}_{k \epsilon}(\boldsymbol{r}),
$$


where $\boldsymbol{\psi}_{k \epsilon}$ is the solution of Maxwell equations "generated" by a plane wave with wave vector $\boldsymbol{k}$ and polarization $\epsilon$. It can be explicitly written as

$$
\begin{aligned}
\boldsymbol{\psi}_{k \epsilon}(\boldsymbol{r}) & =\rho_{\epsilon}(\boldsymbol{k}) \sum_{j=-N_{\mathrm{L}}}^{N_{\mathrm{R}}} \chi_{j}(z) \\
\times & \left(u_{j k \epsilon} \boldsymbol{e}_{k^{(j)} \epsilon} \exp \left(\mathrm{i} \boldsymbol{k}^{(j)} \boldsymbol{r}^{(j)}\right)+v_{j k_{*} \epsilon} \boldsymbol{e}_{k_{*}^{(j)} \epsilon} \exp \left(\mathrm{i} \boldsymbol{k}_{*}^{(j)} \boldsymbol{r}^{(j)}\right)\right),
\end{aligned}
$$

where $\boldsymbol{e}_{k \epsilon}$ is the polarization versor. Coefficients $u_{j k \epsilon}$ and $v_{j k \epsilon}$ in the above expression relate the amplitudes of plane waves in layers of the structure (their definitions have been recalled in Appendix). Equation (11) can be written in a more compact form

$$
\boldsymbol{\psi}_{k \epsilon}(\boldsymbol{r})=\rho_{\epsilon}(\boldsymbol{k}) \mathrm{e}^{\mathrm{i} k_{x} x} \mathrm{e}^{\mathrm{i} k_{y} y} \boldsymbol{\phi}_{k \epsilon}(z)
$$

where

$$
\begin{aligned}
\phi_{k \epsilon}(z) & =\sum_{j=-N_{\mathrm{L}}}^{N_{\mathrm{R}}} \chi_{j}(z) \\
& \times\left(u_{j k \epsilon} e_{k^{(j)} \epsilon} \exp \left(\mathrm{i} k_{z}^{(j)} z^{(j)}\right)+v_{j k_{*} \epsilon} e_{k_{*}^{(j)} \epsilon} \exp \left(-\mathrm{i} k_{z}^{(j)} z^{(j)}\right)\right) .
\end{aligned}
$$

Modes $\boldsymbol{\psi}_{k \epsilon}$ form an independent set which can be transformed into a set of orthonormal modes $\boldsymbol{f}_{k \epsilon}$. Modes $\boldsymbol{f}_{k \epsilon}$ satisfy the same wave equation as $\boldsymbol{\psi}_{k \epsilon}$ and, as pointed out in [7], their orthonormalization rule is

$$
\int \mathrm{d}^{3} r n^{2}(\boldsymbol{r}) \boldsymbol{f}_{q \lambda}^{*}(\boldsymbol{r}) \boldsymbol{f}_{k \epsilon}(\boldsymbol{r})=\delta_{\epsilon \lambda} \delta(\boldsymbol{k}-\boldsymbol{q})
$$

( $\boldsymbol{q}$ and $\lambda$ denote the wave vector and the polarization of a mode, respectively), which allows a straightforward calculation of energy of electric field.

If a similar integral for modes $\boldsymbol{\psi}_{k \epsilon}$ was calculated, then the relation between the two sets would be found easily. Using (12), the integral becomes

$$
\begin{aligned}
\int \mathrm{d}^{3} r n^{2}(\boldsymbol{r}) \boldsymbol{\psi}_{q \lambda}^{*}(\boldsymbol{r}) \boldsymbol{\psi}_{k \epsilon}(\boldsymbol{r})=4 \pi^{2} \rho_{\epsilon}(\boldsymbol{k}) \rho_{\lambda}(\boldsymbol{q}) \\
\quad \times \delta\left(k_{x}-q_{x}\right) \delta\left(k_{y}-q_{y}\right) \int_{-\infty}^{\infty} \mathrm{d} z n^{2}(z) \boldsymbol{\phi}_{q \lambda}^{*}(z) \boldsymbol{\phi}_{k \epsilon}(z),
\end{aligned}
$$

because the integrals over $x$ and $y$ lead to the delta functions. Their presence makes the calculation of the remaining integral over $z$ much easier - it is enough to perform the integration for $q_{x}=k_{x}$ and $q_{y}=k_{y}$, thus, consequently, also $q_{\|}=k_{\|}$. However, in this case both wave vectors $\boldsymbol{k}$ and $\boldsymbol{q}$ define the same plane of incidence. Therefore, if one of the fields has polarization TE and the second field has polarization TM, the first is perpendicular to the plane of incidence, while the second lies within the plane of incidence, which means that they are perpendicular to each other and their scalar product is always zero. For this reason, integral (15) can be simplified

$$
\int \mathrm{d}^{3} r n^{2}(\boldsymbol{r}) \boldsymbol{\psi}_{q \lambda}^{*}(\boldsymbol{r}) \boldsymbol{\psi}_{k \epsilon}(\boldsymbol{r})=4 \pi^{2} \rho_{\epsilon}(\boldsymbol{k}) \rho_{\epsilon}(\boldsymbol{q})
$$




$$
\times \delta\left(k_{x}-q_{x}\right) \delta\left(k_{y}-q_{y}\right) \delta_{\epsilon \lambda} \int_{-\infty}^{\infty} \mathrm{d} z n^{2}(z) \phi_{q \epsilon}^{*}(z) \phi_{k \epsilon}(z) .
$$

The integral over $z$ can be explicitly written as

$$
\begin{aligned}
& \int_{-\infty}^{\infty} \mathrm{d} z n^{2}(z) \boldsymbol{\phi}_{q \epsilon}^{*}(z) \phi_{k \epsilon}(z)=\sum_{j=-N_{\mathrm{L}}}^{N_{\mathrm{R}}} n_{(j)}^{2} \\
& \quad \times \int_{(j)} \mathrm{d} \zeta\left(u_{j q \epsilon} \boldsymbol{e}_{q^{(j)} \epsilon} \exp \left(\mathrm{i} q_{z}^{(j)} \zeta\right)+v_{j q_{*} \epsilon} \boldsymbol{e}_{q_{*}^{(j)} \epsilon} \exp \left(-\mathrm{i} q_{z}^{(j)} \zeta\right)\right)^{*} \\
& \quad \times\left(u_{j k \epsilon} \boldsymbol{e}_{k^{(j)} \epsilon} \exp \left(\mathrm{i} k_{z}^{(j)} \zeta\right)+v_{j k_{*} \epsilon} \boldsymbol{e}_{k_{*}^{(j)} \epsilon} \exp \left(-\mathrm{i} k_{z}^{(j)} \zeta\right)\right)
\end{aligned}
$$

where $\int_{(j)}$ denotes integration in the local coordinate system of the $j$-th layer. Coefficients $u_{j k \epsilon}$ and $v_{j k \epsilon}$ satisfy the following relations:

$$
u_{j k_{*} \epsilon}=u_{j k \epsilon}^{*}, \quad v_{j k_{*} \epsilon}=v_{j k \epsilon}^{*},
$$

and it is very easy to check that

$$
\boldsymbol{e}_{q_{*} \epsilon}^{*} \boldsymbol{e}_{k_{*} \epsilon}=\boldsymbol{e}_{q \epsilon}^{*} \boldsymbol{e}_{k \epsilon}, \quad \boldsymbol{e}_{q_{*} \epsilon}^{*} \boldsymbol{e}_{k \epsilon}=\boldsymbol{e}_{q \epsilon}^{*} \boldsymbol{e}_{k_{*} \epsilon}
$$

(e.g. using the explicit formulae (A.1) and (A.2) from Appendix). Therefore, after carrying out of the integrals, Eq. (17) becomes

$$
\begin{aligned}
& \int_{-\infty}^{\infty} \mathrm{d} z n^{2}(z) \boldsymbol{\phi}_{q \epsilon}^{*}(z) \boldsymbol{\phi}_{k \epsilon}(z)=-\mathrm{i} \sum_{j=-N_{\mathrm{L}}}^{N_{\mathrm{R}}} n_{(j)}^{2}\left(\boldsymbol{e}_{q^{(j)} \epsilon}^{*} \boldsymbol{e}_{k^{(j)} \epsilon}\right. \\
& \quad \times\left.\frac{u_{j q \epsilon}^{*} u_{j k \epsilon} \exp \left(\mathrm{i}\left(k_{z}^{(j)}-q_{z}^{(j) *}\right) \zeta\right)-v_{j q \epsilon} v_{j k \epsilon}^{*} \exp \left(-\mathrm{i}\left(k_{z}^{(j)}-q_{z}^{(j) *}\right) \zeta\right)}{k_{z}^{(j)}-q_{z}^{(j) *}}\right|_{\partial(j)} \\
& \quad+\boldsymbol{e}_{q_{*}^{*}{ }^{(j)} \epsilon} \boldsymbol{e}_{k^{(j)} \epsilon} \\
& \left.\times\left.\frac{v_{j q \epsilon} u_{j k \epsilon} \exp \left(\mathrm{i}\left(k_{z}^{(j)}+q_{z}^{(j) *}\right) \zeta\right)-u_{j q \epsilon}^{*} v_{j k \epsilon}^{*} \exp \left(-\mathrm{i}\left(k_{z}^{(j)}+q_{z}^{(j) *}\right) \zeta\right)}{k_{z}^{(j)}+q_{z}^{(j) *}}\right|_{\partial(j)}\right),
\end{aligned}
$$

where $\left.\right|_{\partial(j)}$ means the difference of values of the term at the $j$-th layer's boundaries. The term resulting from evaluation at the lower boundary of the $(j+1)$-th layer (which is $\zeta=0$ ) cancels out with the term resulting from evaluation at the upper boundary of the $j$-th layer $\left(L_{z}^{(j)}\right.$ for $j>-N_{\mathrm{L}}$ and 0 for $\left.j=-N_{\mathrm{L}}\right)$. Indeed, using relations (A.17) and (A.18) one obtains

$$
\begin{aligned}
& u_{(j+1) q \epsilon}^{*} u_{(j+1) k \epsilon}-v_{(j+1) q \epsilon} v_{(j+1) k \epsilon}^{*}=\frac{1}{2}\left[\left(k_{z}^{(j)} / k_{z}^{(j+1)}+q_{z}^{(j) *} / q_{z}^{(j+1) *}\right)\right. \\
& \quad \times\left(u_{j q \epsilon}^{*} u_{j k \epsilon} \exp \left(\mathrm{i}\left(k_{z}^{(j)}-q_{z}^{(j) *}\right) L_{z}^{(j)}\right)-v_{j q \epsilon} v_{j k \epsilon}^{*} \exp \left(-\mathrm{i}\left(k_{z}^{(j)}-q_{z}^{(j) *}\right) L_{z}^{(j)}\right)\right) \\
& \quad+\left(k_{z}^{(j)} / k_{z}^{(j+1)}-q_{z}^{(j) *} / q_{z}^{(j+1) *}\right) \\
& \left.\times\left(v_{j q \epsilon} u_{j k \epsilon} \exp \left(\mathrm{i}\left(k_{z}^{(j)}+q_{z}^{(j) *}\right) L_{z}^{(j)}\right)-u_{j q \epsilon}^{*} v_{j k \epsilon}^{*} \exp \left(-\mathrm{i}\left(k_{z}^{(j)}+q_{z}^{(j) *}\right) L_{z}^{(j)}\right)\right)\right],(21)
\end{aligned}
$$




$$
\begin{aligned}
& v_{(j+1) q \epsilon} u_{(j+1) k \epsilon}-u_{(j+1) q \epsilon}^{*} v_{(j+1) k \epsilon}^{*}=\frac{1}{2}\left[\left(k_{z}^{(j)} / k_{z}^{(j+1)}-q_{z}^{(j) *} / q_{z}^{(j+1) *}\right)\right. \\
& \quad \times\left(u_{j q \epsilon}^{*} u_{j k \epsilon} \exp \left(\mathrm{i}\left(k_{z}^{(j)}-q_{z}^{(j) *}\right) L_{z}^{(j)}\right)-v_{j q \epsilon} v_{j k \epsilon}^{*} \exp \left(-\mathrm{i}\left(k_{z}^{(j)}-q_{z}^{(j) *}\right) L_{z}^{(j)}\right)\right) \\
& \quad+\left(k_{z}^{(j)} / k_{z}^{(j+1)}+q_{z}^{(j) *} / q_{z}^{(j+1) *}\right) \\
& \left.\times\left(v_{j q \epsilon} u_{j k \epsilon} \exp \left(\mathrm{i}\left(k_{z}^{(j)}+q_{z}^{(j) *}\right) L_{z}^{(j)}\right)-u_{j q \epsilon}^{*} v_{j k \epsilon}^{*} \exp \left(-\mathrm{i}\left(k_{z}^{(j)}+q_{z}^{(j) *}\right) L_{z}^{(j)}\right)\right)\right](22)
\end{aligned}
$$

and after a simple, but lengthy calculation (in which (A.5) is useful) it turns out that the terms are equal, but they appear in (20) with opposite signs.

The remaining terms arise from the limits in $\pm \infty$. To make the formulae more elegant, let $\mathrm{R} \equiv N_{\mathrm{R}}$ and $\mathrm{L} \equiv-N_{\mathrm{L}}$. For radiation modes all the $z$ components in $j=\mathrm{R}$ and $j=\mathrm{L}$ regions are real: $k_{z}^{(\mathrm{R})}, k_{z}^{(\mathrm{L})}, q_{z}^{(\mathrm{R})}, q_{z}^{(\mathrm{L})} \in \mathcal{R}$, and expression (20) is then

$$
\begin{aligned}
\int_{-\infty}^{\infty} & \mathrm{d} z n^{2}(z) \boldsymbol{\phi}_{q \epsilon}^{*}(z) \phi_{k \epsilon}(z)=-\mathrm{i} \lim _{\zeta \rightarrow \infty}\left[n _ { ( \mathrm { R } ) } ^ { 2 } \left(\boldsymbol{e}_{q^{(\mathrm{R})} \epsilon}^{*} \boldsymbol{e}_{k^{(\mathrm{R})} \epsilon}\right.\right. \\
& \times \frac{u_{\mathrm{R} q \epsilon}^{*} u_{\mathrm{R} k \epsilon} \exp \left(\mathrm{i}\left(k_{z}^{(\mathrm{R})}-q_{z}^{(\mathrm{R})}\right) \zeta\right)-v_{\mathrm{R} q \epsilon} v_{\mathrm{R} k \epsilon}^{*} \exp \left(-\mathrm{i}\left(k_{z}^{(\mathrm{R})}-q_{z}^{(\mathrm{R})}\right) \zeta\right)}{k_{z}^{(\mathrm{R})}-q_{z}^{(\mathrm{R})}} \\
& +\boldsymbol{e}_{q_{*}^{*}{ }^{(\mathrm{R})} \epsilon} \boldsymbol{e}_{k^{(\mathrm{R})} \epsilon} \\
& \left.\times \frac{v_{\mathrm{R} q \epsilon} u_{\mathrm{R} k \epsilon} \exp \left(\mathrm{i}\left(k_{z}^{(\mathrm{R})}+q_{z}^{(\mathrm{R})}\right) \zeta\right)-u_{\mathrm{R} q \epsilon}^{*} v_{\mathrm{R} k \epsilon}^{*} \exp \left(-\mathrm{i}\left(k_{z}^{(\mathrm{R})}+q_{z}^{(\mathrm{R})}\right) \zeta\right)}{k_{z}^{(\mathrm{R})}+q_{z}^{(\mathrm{R})}}\right) \\
& -n_{(\mathrm{L})}^{2}\left(\boldsymbol{e}_{q^{(\mathrm{L})} \epsilon}^{*} \boldsymbol{e}_{k^{(\mathrm{L})} \epsilon}\right. \\
& \times \frac{u_{\mathrm{L} q \epsilon}^{*} u_{\mathrm{L} k \epsilon} \exp \left(-\mathrm{i}\left(k_{z}^{(\mathrm{L})}-q_{z}^{(\mathrm{L})}\right) \zeta\right)-v_{\mathrm{L} q \epsilon} v_{\mathrm{L} k \epsilon}^{*} \exp \left(\mathrm{i}\left(k_{z}^{(\mathrm{L})}-q_{z}^{(\mathrm{L})}\right) \zeta\right)}{k_{z}^{(\mathrm{L})}-q_{z}^{(\mathrm{L})}} \\
& +\boldsymbol{e}_{q_{*}^{(\mathrm{L})} \epsilon}^{*} \boldsymbol{e}_{k(\mathrm{~L}) \epsilon} \\
& \left.\left.\times \frac{v_{\mathrm{L} q \epsilon} u_{\mathrm{L} k \epsilon} \exp \left(-\mathrm{i}\left(k_{z}^{(\mathrm{L})}+q_{z}^{(\mathrm{L})}\right) \zeta\right)-u_{\mathrm{L} q \epsilon}^{*} v_{\mathrm{L} k \epsilon}^{*} \exp \left(\mathrm{i}\left(k_{z}^{(\mathrm{L})}+q_{z}^{(\mathrm{L})}\right) \zeta\right)}{k_{z}^{(\mathrm{L})}+q_{z}^{(\mathrm{L})}}\right)\right] .
\end{aligned}
$$

An exponent can be split into a sum of cosine and sine functions, therefore

$$
\lim _{\zeta \rightarrow \infty} \frac{\mathrm{e}^{\mathrm{i} k \zeta}}{k}=\lim _{\zeta \rightarrow \infty} \frac{\cos (k \zeta)}{k}+\mathrm{i} \lim _{\zeta \rightarrow \infty} \frac{\sin (k \zeta)}{k} .
$$

The term containing the cosine function can be omitted $[1,3]$, while the term with the sine gives

$$
\lim _{\zeta \rightarrow \infty} \frac{\sin (k \zeta)}{k}=\pi \delta(k) .
$$

In the presence of the arising delta functions all the polarization versors in (23) become either $\boldsymbol{e}_{k^{(\mathrm{R})} \epsilon}$ or $\boldsymbol{e}_{k^{(\mathrm{L})} \epsilon}$, and all their scalar products become equal to 1 . The calculated integral is then 


$$
\begin{aligned}
& \int_{-\infty}^{\infty} \mathrm{d} z n^{2}(z) \phi_{q \epsilon}^{*}(z) \phi_{k \epsilon}(z)=\pi\left\{n _ { ( \mathrm { R } ) } ^ { 2 } \left[\left(\left|u_{\mathrm{R} k \epsilon}\right|^{2}+\left|v_{\mathrm{R} k \epsilon}\right|^{2}\right) \delta\left(k_{z}^{(\mathrm{R})}-q_{z}^{(\mathrm{R})}\right)\right.\right. \\
& \left.\quad+2 u_{\mathrm{R} k \epsilon} v_{\mathrm{R} k \epsilon}^{*} \delta\left(k_{z}^{(\mathrm{R})}+q_{z}^{(\mathrm{R})}\right)\right]+n_{(\mathrm{L})}^{2}\left[\left(\left|u_{\mathrm{L} k \epsilon}\right|^{2}+\left|v_{\mathrm{L} k \epsilon}\right|^{2}\right) \delta\left(k_{z}^{(\mathrm{L})}-q_{z}^{(\mathrm{L})}\right)\right. \\
& \left.\left.\quad+2 u_{\mathrm{L} k \epsilon} v_{\mathrm{L} k \epsilon}^{*} \delta\left(k_{z}^{(\mathrm{L})}+q_{z}^{(\mathrm{L})}\right)\right]\right\} .
\end{aligned}
$$

Using the well-known property of the delta function

$$
\delta(f(x))=\sum_{i} \delta\left(x-x_{i}\right) /\left|\frac{\mathrm{d} f}{\mathrm{~d} x}\right|, \quad x_{i}: f\left(x_{i}\right)=0,
$$

one obtains

$$
\delta\left(k_{z}^{(j)} \mp q_{z}^{(j)}\right)=\frac{n_{(0)}^{2}}{n_{(j)}^{2}}\left|\frac{k_{z}^{(j)}}{k_{z}}\right| \delta\left(k_{z} \mp q_{z}\right) .
$$

Finally

$$
\begin{aligned}
& \int_{-\infty}^{\infty} \mathrm{d} z n^{2}(z) \phi_{q \epsilon}^{*}(z) \phi_{k \epsilon}(z) \\
& =\pi n_{(0)}^{2}\left\{\left|\frac{k_{z}^{(\mathrm{R})}}{k_{z}}\right|\left[\left(\left|u_{\mathrm{R} k \epsilon}\right|^{2}+\left|v_{\mathrm{R} k \epsilon}\right|^{2}\right) \delta\left(k_{z}-q_{z}\right)+2 u_{\mathrm{R} k \epsilon} v_{\mathrm{R} k \epsilon}^{*} \delta\left(k_{z}+q_{z}\right)\right]\right. \\
& \left.+\left|\frac{k_{z}^{(\mathrm{L})}}{k_{z}}\right|\left[\left(\left|u_{\mathrm{L} k \epsilon}\right|^{2}+\left|v_{\mathrm{L} k \epsilon}\right|^{2}\right) \delta\left(k_{z}-q_{z}\right)+2 u_{\mathrm{L} k \epsilon} v_{\mathrm{L} k \epsilon}^{*} \delta\left(k_{z}+q_{z}\right)\right]\right\}
\end{aligned}
$$

The obtained result lets to write the integral (15) as

$$
\int \mathrm{d}^{3} r n^{2}(\boldsymbol{r}) \boldsymbol{\psi}_{q \lambda}^{*}(\boldsymbol{r}) \boldsymbol{\psi}_{k \epsilon}(\boldsymbol{r})=\rho_{\epsilon}^{2}(\boldsymbol{k}) \delta_{\epsilon \lambda}\left[F_{k \epsilon} \delta(\boldsymbol{k}-\boldsymbol{q})+\tilde{F}_{k \epsilon} \delta\left(\boldsymbol{k}-\boldsymbol{q}_{*}\right)\right],
$$

where

$$
\begin{aligned}
& F_{k \epsilon}=4 \pi^{3} n_{(0)}^{2} \frac{\left|k_{z}^{(\mathrm{R})}\right|\left(\left|u_{\mathrm{R} k \epsilon}\right|^{2}+\left|v_{\mathrm{R} k \epsilon}\right|^{2}\right)+\left|k_{z}^{(\mathrm{L})}\right|\left(\left|u_{\mathrm{L} k \epsilon}\right|^{2}+\left|v_{\mathrm{L} k \epsilon}\right|^{2}\right)}{\left|k_{z}\right|}, \\
& \tilde{F}_{k \epsilon}=8 \pi^{3} n_{(0)}^{2} \frac{\left|k_{z}^{(\mathrm{R})}\right| u_{\mathrm{R} k \epsilon} v_{\mathrm{R} k \epsilon}^{*}+\left|k_{z}^{(\mathrm{L})}\right| u_{\mathrm{L} k \epsilon} v_{\mathrm{L} k \epsilon}^{*}}{\left|k_{z}\right|} .
\end{aligned}
$$

The right hand side of (30) suggests that the relation between $\boldsymbol{\psi}_{k \epsilon}$ and $\boldsymbol{f}_{k \epsilon}$ has the form

$$
\boldsymbol{\psi}_{k \epsilon}(\boldsymbol{r})=r_{k \epsilon}\left(\boldsymbol{f}_{k \epsilon}(\boldsymbol{r})+s_{k \epsilon} \boldsymbol{f}_{k_{*} \epsilon}(\boldsymbol{r})\right) .
$$

The calculation of (30), with the use of (14), gives

$$
r_{k \epsilon}=\rho_{\epsilon}(\boldsymbol{k}) \sqrt{\frac{F_{k \epsilon}}{1+\left|s_{k \epsilon}\right|^{2}}},
$$




$$
s_{k \epsilon}= \begin{cases}\frac{F_{k \epsilon}-\sqrt{F_{k \epsilon}^{2}-\left|\tilde{F}_{k \epsilon}\right|^{2}}}{\tilde{F}_{k \epsilon}}, & \tilde{F}_{k \epsilon} \neq 0, \\ 0, & \tilde{F}_{k \epsilon}=0 .\end{cases}
$$

Hence, the orthonormal electric radiation modes are

$$
\boldsymbol{f}_{k \epsilon}(\boldsymbol{r})=\frac{1}{r_{k \epsilon}} \frac{\boldsymbol{\psi}_{k \epsilon}(\boldsymbol{r})-s_{k \epsilon} \boldsymbol{\psi}_{k_{*} \epsilon}(\boldsymbol{r})}{1-\left|s_{k \epsilon}\right|^{2}}
$$

or, written in a more explicit form

$$
\begin{aligned}
& \boldsymbol{f}_{k \epsilon}(\boldsymbol{r})=\frac{1}{1-\left|s_{k \epsilon}\right|^{2}} \sqrt{\frac{1+\left|s_{k \epsilon}\right|^{2}}{F_{k \epsilon}}} \sum_{j=-N_{\mathrm{L}}}^{N_{\mathrm{R}}} \chi_{j}(z)\left[\left(u_{j k \epsilon}-s_{k \epsilon} v_{j k \epsilon}\right) \boldsymbol{e}_{k(j)} \epsilon\right. \\
& \left.\quad \times \exp \left(\mathrm{i} \boldsymbol{k}^{(j)} \boldsymbol{r}^{(j)}\right)+\left(v_{j k_{*} \epsilon}-s_{k \epsilon} u_{j k_{*} \epsilon}\right) \boldsymbol{e}_{k_{*}^{(j)} \epsilon} \exp \left(\mathrm{i} \boldsymbol{k}_{*}^{(j)} \boldsymbol{r}^{(j)}\right)\right] .
\end{aligned}
$$

\section{Magnetic field}

In every layer of the considered structure, magnetic field is related to electric field by the expression obtained from the Maxwell equations for a superposition of two plane waves with the same frequency

$$
\boldsymbol{\psi}_{k \epsilon}^{H}(\boldsymbol{r})=\frac{\nabla \times \boldsymbol{\psi}_{k \epsilon}(\boldsymbol{r})}{\mathrm{i} \mu_{0} \omega_{k}}
$$

With the definition of polarization versors of magnetic field

$$
\boldsymbol{e}_{k \epsilon}^{H}=\frac{\boldsymbol{k} \times \boldsymbol{e}_{k \epsilon}}{k},
$$

the distribution of the field is given by

$$
\begin{aligned}
& \boldsymbol{\psi}_{k \epsilon}^{H}(\boldsymbol{r})=\frac{\rho_{\epsilon}(\boldsymbol{k})}{\mu_{0} c} \sum_{j=-N_{\mathrm{L}}}^{N_{\mathrm{R}}} \chi_{j}(z) n_{(j)}\left(u_{j k \epsilon} \boldsymbol{e}_{k^{(j)} \epsilon}^{H} \exp \left(\mathrm{i} \boldsymbol{k}^{(j)} \boldsymbol{r}^{(j)}\right)\right. \\
& \left.\quad+v_{j k_{*} \epsilon} \boldsymbol{e}_{k_{*}^{(j)} \epsilon}^{H} \exp \left(\mathrm{i} \boldsymbol{k}_{*}^{(j)} \boldsymbol{r}^{(j)}\right)\right) .
\end{aligned}
$$

If the versors (39) are calculated with the use of (A.1) and (A.2), it turns out that

$$
\begin{aligned}
& \boldsymbol{e}_{k \mathrm{TE}}^{H}=-\boldsymbol{e}_{k \mathrm{TM}}, \\
& \boldsymbol{e}_{k \mathrm{TM}}^{H}=\boldsymbol{e}_{k \mathrm{TE}} .
\end{aligned}
$$

Because refractive index is already incorporated in (40), it can be easily seen that the integral, which arises by calculation of energy of magnetic field, is proportional to already calculated integral for electric field

$$
\int \mathrm{d}^{3} r\left(\boldsymbol{\psi}_{q \lambda}^{H}(\boldsymbol{r})\right)^{*} \boldsymbol{\psi}_{k \epsilon}^{H}(\boldsymbol{r})=\frac{\varepsilon_{0}}{\mu_{0}} \int \mathrm{d}^{3} r n^{2}(\boldsymbol{r}) \boldsymbol{\psi}_{q(-\lambda)}^{*}(\boldsymbol{r}) \boldsymbol{\psi}_{k(-\epsilon)}(\boldsymbol{r}),
$$

where the notation $(-\epsilon)$ means "the other polarization", i.e. $(-\mathrm{TE})=\mathrm{TM}$ and $(-\mathrm{TM})=\mathrm{TE}$. It is worth noting that because the above conclusion has been reached by considering only fundamental relations between electric and magnetic fields, (43) is valid not only for radiation modes, but for all modes of the structure. A similar relation is satisfied by 
$\boldsymbol{f}_{k \epsilon}^{H}(\boldsymbol{r})=\frac{\nabla \times \boldsymbol{f}_{k \epsilon}(\boldsymbol{r})}{\mathrm{i} \mu_{0} \omega_{k}}$

\section{Appendix}

\section{A few formulae and calculations}

Explicit expressions for electric field polarization versors are

$$
\begin{aligned}
\boldsymbol{e}_{k \mathrm{TE}} & =\frac{k_{y} \boldsymbol{e}_{x}-k_{x} \boldsymbol{e}_{y}}{k_{\|}}, \\
\boldsymbol{e}_{k \mathrm{TM}} & =\frac{-k_{x} k_{z} \boldsymbol{e}_{x}-k_{y} k_{z} \boldsymbol{e}_{y}+k_{\|}^{2} \boldsymbol{e}_{z}}{|\boldsymbol{k}| k_{\|}} .
\end{aligned}
$$

For $q_{x}=k_{x}$ and $q_{y}=k_{y}$ :

$$
\begin{aligned}
& \boldsymbol{e}_{q \mathrm{TE}}^{*} \boldsymbol{e}_{k \mathrm{TE}}=1, \\
& \boldsymbol{e}_{q \mathrm{TM}}^{*} \boldsymbol{e}_{k \mathrm{TM}}=\frac{k_{z} q_{z}^{*}+k_{\|}^{2}}{|\boldsymbol{k} \| \boldsymbol{q}|}
\end{aligned}
$$

and, from (7)

$$
\left(k_{z}^{(j)}\right)^{2}-\left(q_{z}^{(j) *}\right)^{2}=\left(k_{z}^{(j)}\right)^{2}-\left(q_{z}^{(j)}\right)^{2}=\frac{n_{(j)}^{2}}{c^{2}}\left(\omega_{k}^{2}-\omega_{q}^{2}\right) .
$$

The matrix $m_{j, 0}$, which, for $j>0$, establishes the relation between amplitudes of waves in the layer $j=0$ (containing the radiation source) and in the $j$-th layer, allows to define the coefficients $[6]$ :

$$
\begin{aligned}
& u_{j k \epsilon}=\left(\delta_{\epsilon \mathrm{TE}}+\frac{n_{(0)}}{n_{(j)}} \delta_{\epsilon \mathrm{TM}}\right)\left[\left(m_{j, 0}^{\epsilon}(\boldsymbol{k})\right)_{11}+\left(m_{j, 0}^{\epsilon}(\boldsymbol{k})\right)_{12} \xi_{\epsilon}^{*}(\boldsymbol{k})\right], \\
& v_{j k \epsilon}=\left(\delta_{\epsilon \mathrm{TE}}+\frac{n_{(0)}}{n_{(j)}} \delta_{\epsilon \mathrm{TM}}\right)\left[\left(m_{j, 0}^{\epsilon}\left(\boldsymbol{k}_{*}\right)\right)_{21}+\left(m_{j, 0}^{\epsilon}\left(\boldsymbol{k}_{*}\right)\right)_{22} \xi_{\epsilon}(\boldsymbol{k})\right],
\end{aligned}
$$

where $\xi_{\epsilon}(\boldsymbol{k})$ is a function defining the relation between waves propagating in opposite $z$ directions. For $j<0$ the relation is established by matrix $w_{j, 0}$

$$
w_{j, 0}=m_{0, j}^{-1}
$$

(keeping in mind that for $j<0$ an imaginary part of $k_{z}^{(j)}$ is negative). Coefficients $u_{j k \epsilon}$ and $v_{j k \epsilon}$ are in this case

$$
\begin{aligned}
& u_{j k \epsilon}=\left(\delta_{\epsilon \mathrm{TE}}+\frac{n_{(0)}}{n_{(j)}} \delta_{\epsilon \mathrm{TM}}\right)\left[\left(w_{j, 0}^{\epsilon}(\boldsymbol{k})\right)_{11}+\left(w_{j, 0}^{\epsilon}(\boldsymbol{k})\right)_{12} \xi_{\epsilon}^{*}(\boldsymbol{k})\right], \\
& v_{j k \epsilon}=\left(\delta_{\epsilon \mathrm{TE}}+\frac{n_{(0)}}{n_{(j)}} \delta_{\epsilon \mathrm{TM}}\right)\left[\left(w_{j, 0}^{\epsilon}\left(\boldsymbol{k}_{*}\right)\right)_{21}+\left(w_{j, 0}^{\epsilon}\left(\boldsymbol{k}_{*}\right)\right)_{22} \xi_{\epsilon}(\boldsymbol{k})\right] .
\end{aligned}
$$

In both these cases, if the matrix $m_{j, 0}$ is expressed simply by

$$
m_{j, 0}=\left(\begin{array}{ll}
m_{11} & m_{12} \\
m_{21} & m_{22}
\end{array}\right),
$$

then the definitions of $u_{j k \epsilon}$ and $v_{j k \epsilon}$ can be written as 


$$
\left(\begin{array}{c}
u_{j k \epsilon} \\
v_{j k \epsilon}^{*}
\end{array}\right)=\left(\begin{array}{ll}
m_{11} & m_{12} \\
m_{21} & m_{22}
\end{array}\right)\left(\begin{array}{c}
1 \\
\xi_{\epsilon}^{*}(\boldsymbol{k})
\end{array}\right)
$$

for $j>0$, and the same, with matrix $m_{j, 0}$ replaced by $w_{j, 0}$, for $j<0$.

Matrix $m_{j+1, j}$, which establishes the relation between waves in $j$-th and $(j+1)$-th layer, is given by

$$
m_{j+1, j}=\frac{1}{2}\left(\begin{array}{cc}
K_{j}^{+} \exp \left(\mathrm{i} k_{z}^{(j)} L_{z}^{(j)}\right) & K_{j}^{-} \exp \left(-\mathrm{i} k_{z}^{(j)} L_{z}^{(j)}\right) \\
K_{j}^{-} \exp \left(\mathrm{i} k_{z}^{(j)} L_{z}^{(j)}\right) & K_{j}^{+} \exp \left(-\mathrm{i} k_{z}^{(j)} L_{z}^{(j)}\right)
\end{array}\right),
$$

where coefficients $K_{j}^{+}$and $K_{j}^{-}$depend on polarization

$$
K_{j}^{\mathrm{TE} \pm}=1 \pm \frac{k_{z}^{(j)}}{k_{z}^{(j+1)}}, \quad K_{j}^{\mathrm{TM} \pm}=1 \pm \frac{n_{(j+1)}^{2}}{n_{(j)}^{2}} \frac{k_{z}^{(j)}}{k_{z}^{(j+1)}} .
$$

Matrix $m_{j+1,0}$ is the product of $m_{j+1, j}$ and $m_{j, 0}$ :

$$
m_{j+1,0}=m_{j+1, j} m_{j, 0} \text {. }
$$

Similarly, matrix $w_{j+1,0}$ for $j<0$ :

$$
w_{j+1,0}=m_{j+1, j} w_{j, 0} .
$$

After these two simple calculations, it follows from (A.12) that for every $j$

$$
\begin{gathered}
u_{(j+1) k \epsilon}=\frac{1}{2}\left(\delta_{\epsilon \mathrm{TE}}+\frac{n_{(j)}}{n_{(j+1)}} \delta_{\epsilon \mathrm{TM}}\right) \\
\times\left(K_{j}^{+} u_{j k \epsilon} \exp \left(\mathrm{i} k_{z}^{(j)} L_{z}^{(j)}\right)+K_{j}^{-} v_{j k \epsilon}^{*} \exp \left(-\mathrm{i} k_{z}^{(j)} L_{z}^{(j)}\right)\right) \\
v_{(j+1) k \epsilon}=\frac{1}{2}\left(\delta_{\epsilon \mathrm{TE}}+\frac{n_{(j)}}{n_{(j+1)}} \delta_{\epsilon \mathrm{TM}}\right) \\
\times\left(K_{j}^{-} u_{j k \epsilon} \exp \left(\mathrm{i} k_{z}^{(j)} L_{z}^{(j)}\right)+K_{j}^{+} v_{j k \epsilon}^{*} \exp \left(-\mathrm{i} k_{z}^{(j)} L_{z}^{(j)}\right)\right)^{*} .
\end{gathered}
$$

\section{References}

[1] D. Marcuse, Light Transmission Optics, Van Nostrand-Reinhold, New York 1972, p. 313.

[2] P. Benech, D.A.M. Khalil, F.S. Andre, Opt. Commun. 88, 96 (1992).

[3] S.-L. Lee, D.S.L. Mui, L.A. Coldren, J. Lightwave Technol. 12, 2073 (1994).

[4] H. Rigneault, S. Monneret, Quantum Semiclass. Opt. 9, 1017 (1997).

[5] A. Rudziński, A. Tyszka-Zawadzka, P. Szczepański, Proc. SPIE 5950, 59501A (2005).

[6] A. Rudziński, Acta Phys. Pol. A 111, 323 (2007).

[7] R.J. Glauber, M. Lewenstein, Phys. Rev. A 43, 467 (1991). 